\title{
A Rare Case of Traumatic Ulcerative Granuloma with Stromal Eosinophilia of the Tongue (Tugse) in a Child: Diagnostic Problems and Differential Diagnosis
}

\section{Barca Ida, Cordaro Raffaella, Colangeli Walter, Novembre Daniela, Cristofaro Maria G}

Department of Experimental and Clinical Medicine, Unit of Oral and Maxillofacial Surgery, Magna Graecia University, Viale Europa, Catanzaro, Italy.

\begin{abstract}
Traumatic Ulcerative granuloma with stromal eosinophilia (TUGSE) is an infrequent condition marked by the presence of a solitary ulcer in the oral mucosa. It is generally considered a reactive, benign and self-limiting ulcer with raised and indurated margins, mainly affecting the tongue, cheeks, or, less frequently, lips. His typical histology shows a diffuse polymorphic cell inflammatory infiltrate composed predominantly of eosinophils involving the superficial mucosa and extending deep into the submucosa with degeneration of the underlying muscle layer. Aetiology is unknown and pathogenesis is not clear although trauma could have an important role in the origin and evolution of this lesion. Nevertheless, a correct diagnosis is difficult because the ulcer mimics a wide range of pathologies including microbial infections or cancer.

This work describes a case of traumatic granuloma of the tongue in a child female focusing attention on the differential diagnosis of this unclear lesion
\end{abstract}

Key Words: Eosinophilic granuloma, Reactive benign lesion of the tongue, Traumatic ulcerative granuloma

\section{Introduction}

TUGSE is a reactive benign lesion of oral mucosa with an obscure clinical behavior generally affecting the tongue. Its ulcerative aspect can mimics malignancies or infectious processes but it tends to resolve spontaneously and, in a majority of the cases, trauma is considered to have a central role in the pathogenesis [1]. This lesion affects usually females with a remarkable predilection of the older population especially the black ethnic groups. A bioptic exam of the lesion is crucial to define a TUGSE diagnosis. Histopathological findings are typical and consist of eosinophil-rich mixed infiltrate with small lymphocytes $\mathrm{T}$ and B. In many cases rapid healing is generally achieved spontaneously or after a biopsy; and do not requires any treatment [2]. For clinicians knowledge of this condition is important to guide appropriate patient care and counseling.

We report an uncommon case of TUGSE in a young patient seen at our Department of Oral and Maxillofacial Surgery, University "Magna Graecia” of Catanzaro, Italy.

\section{Case Report}

An 11-year-old female presented herself at the Department of Oral and Maxillo Facial Surgery University Magna Graecia of Catanzaro with non-healing isolated painful ulcer of the tongue for 7 days.

Intraoral examination revealed an ulcer on the dorsal surface and left the side of the tongue measuring $2 \mathrm{~cm} \times 1.5$ $\mathrm{cm}$ in size with well-defined and raised margins surrounded by a small area of erythema, opposite teeth 2.4-2.5-2.6. On palpation, the ulcer was smooth and firm in consistency with indurated margins (Figure 1). No regional lymphadenopathy was present. She gave a history of pain radiating to the cheek and neck region.

Her previous medical history was not remarkable. She referred to an episode of tongue self-bite 2 weeks ago with no improvement of the lesion after medication. No dentoscheletrical or other intraoral anomalies were found.

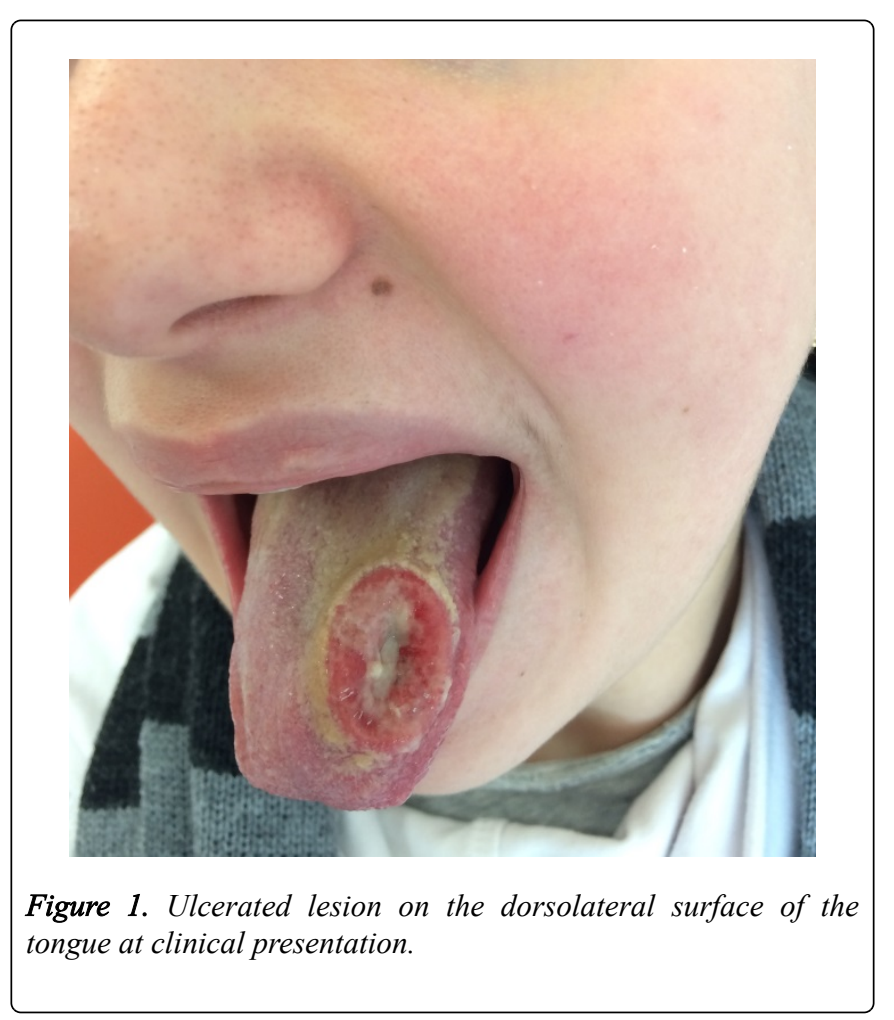

Blood and chemistry tests were performed to exclude hematological or metabolic disorders. Furthermore microbiological and serological tests were also carried out to investigate leishmaniasis, toxoplasmosis, EBV, CMV, Hepatitis B or C, HIV, zygomycosis, aspergillosis, histoplasmosis, blastomycosis, syphilis or autoimmune diseases like Lupus erythematosus, Reiter's syndrome (HLAB27) and Wegener's disease (ANCA). 


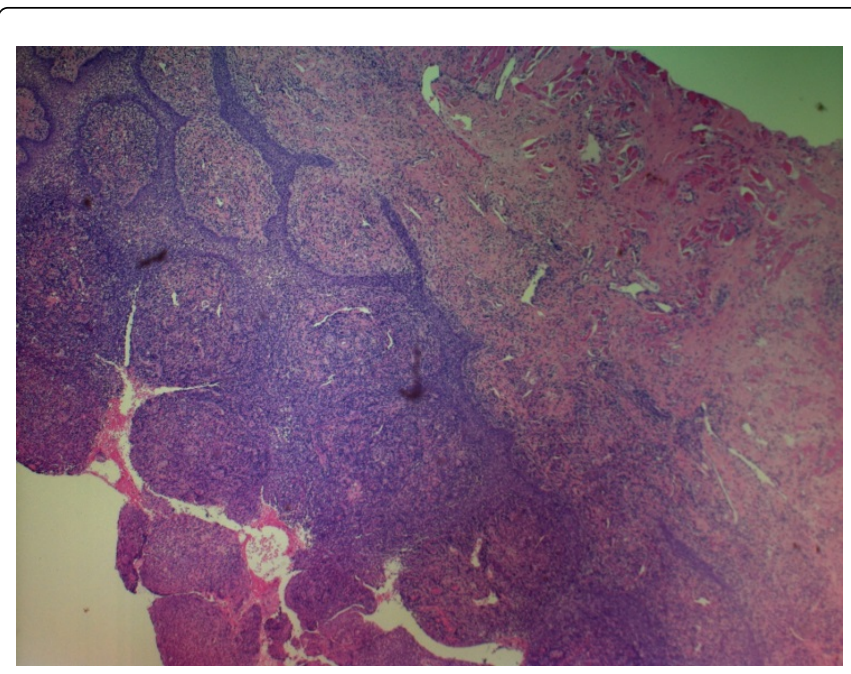

Figure 2. Ulcerated area with mixed inflammatory cells (10X, $\mathrm{H}$ and $E$ ).

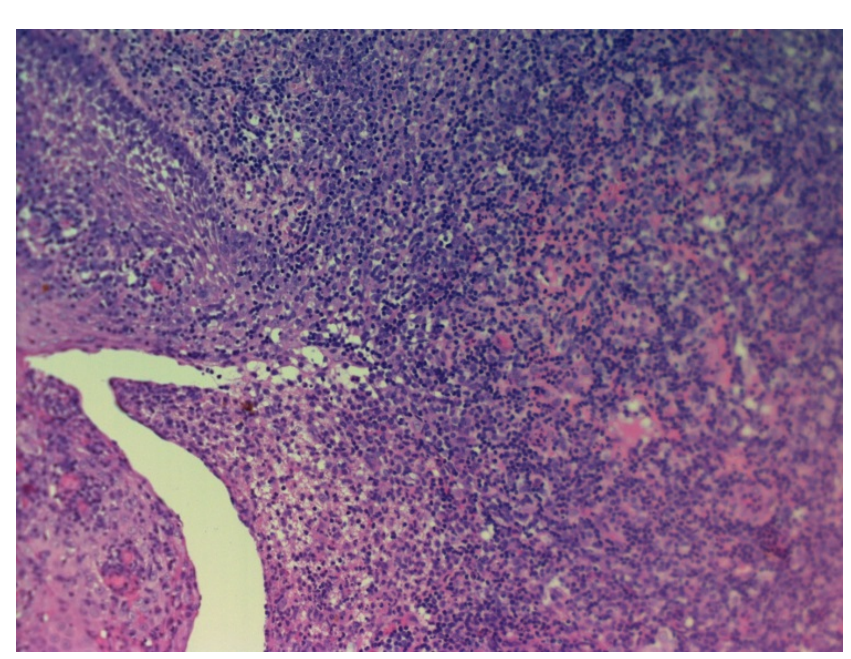

Figure 3. Inflammatory infiltrate of granuloma composed by small lymphocytes $B$ and $T$ and granulocytes (40X, $H$ and $E)$.

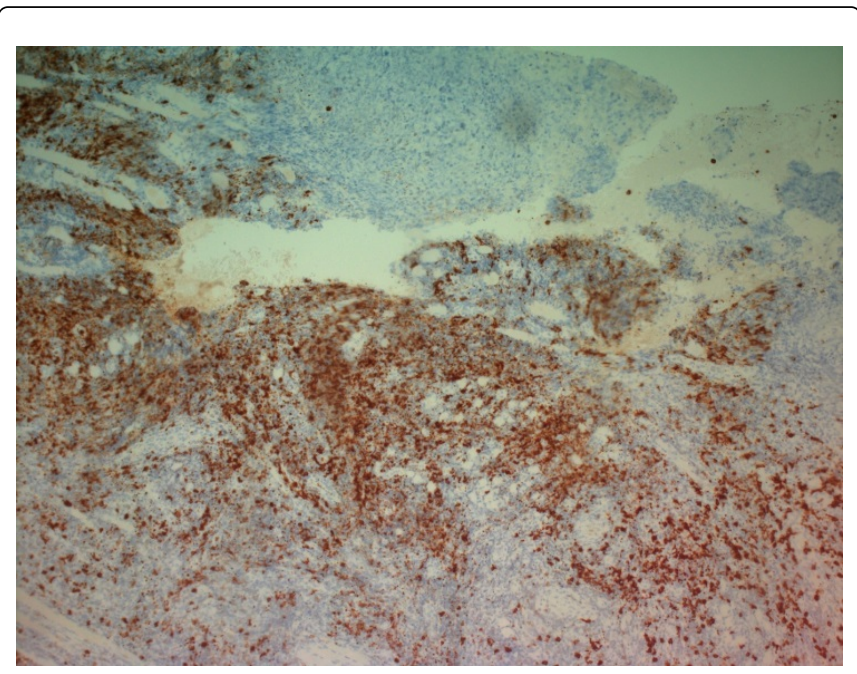

Figure 4. Immunohistochemical analysis showing $C D 3+T$ Lymphocytes aggregates.

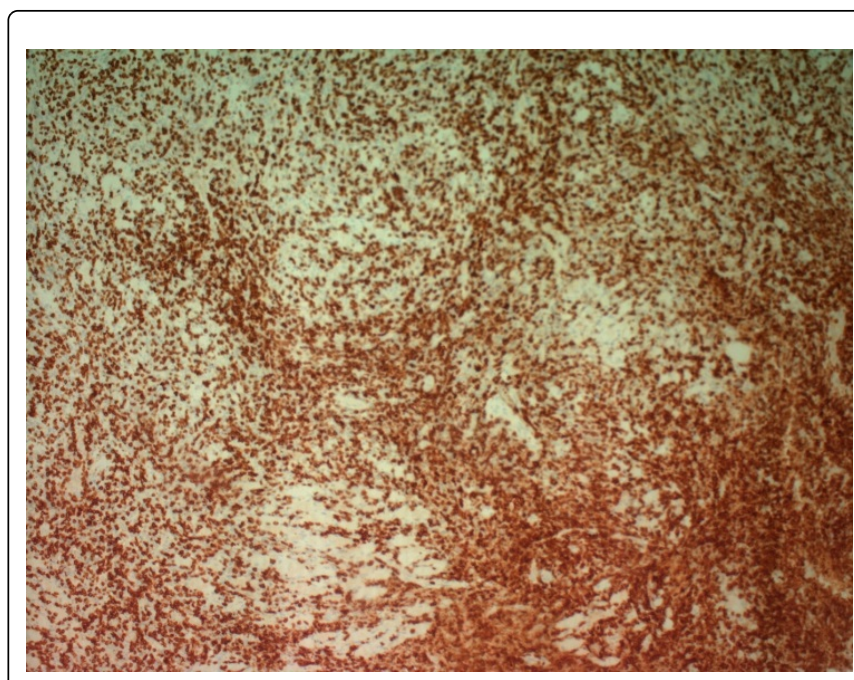

Figure 5. Immunohistochemical analysis showing composed by small lymphocytes $B$ and $T$ and $C D 20+B$ Lymphocytes.

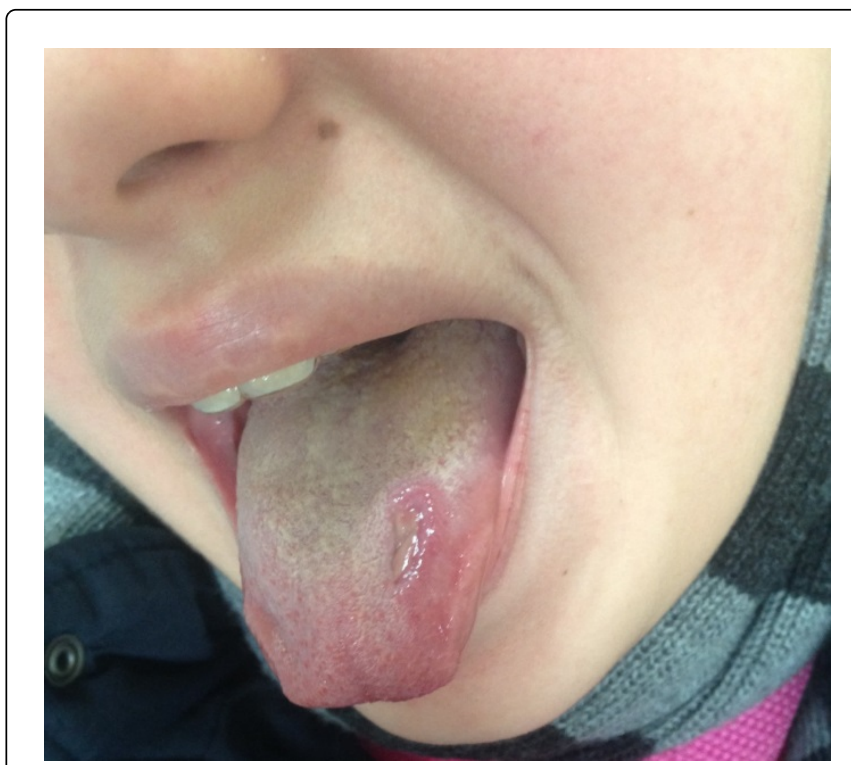

Figure 6. Complete resolution two months post biopsy.

Tuberculosis skin test was added too. Negative results of these laboratories exams excluded a systemic condition and then biopsy was recommended to define the diagnosis. Ultrasonographic exam of Lymph Nodes in Head and Neck showed reactive lymph nodes in the left submandibular region. One week later an incisional biopsy was performed under local anesthesia for histopathological analysis.

Microscopic examination showed superficial hyperplastic epithelium with hyperkeratosis and central area of ulceration. This ulcerated area was infiltrated with mixed inflammatory cells chiefly composed of eosinophils, CD 20+ and CD3+ B and $\mathrm{T}$ lymphocytes, CD68+ histiocytes, CD31+ cells and macrophages extending deep into the muscle layer. The infiltrated tissue was well vascularized. No presence of atypical cells (Figures 2-5).

On the basis of these results, a diagnosis of TUGSE was made. The patient underwent antibiotic therapy with oral Amoxicillin $1 \mathrm{gr}$ for 10 days and was advised to use corticosteroid and chlorhexidine $0.2 \%$ mouthwashes. Then 
she has been monitored closely with routine check-ups. No further treatment was required. Complete healing was noticed after two months with structural and functional resolution (Figure 6).

\section{Discussion}

TUGSE is an infrequent lesion of the oral mucosa with unclear aetiology and pathogenesis. It has been known by different names such as Riga-Fede disease, eosinophilic granuloma, and traumatic granuloma. This lesion was originally described clinically in 1881 by Riga [3] and histologically in 1890 by Fede [4] and identified as a distinct entity in 1970 by Shapiro and Juhlin but only in the 1983, Elzy coined the term TUGSE [5].

Nowadays etiology of TUGSE remains still obscure. The clinical presentation mimics a large range of different pathologies like oral cancer, infectious diseases, metabolic or autoimmune disorders, and aphthous-like lesions. Some authors identified trauma as the main factor in the development of lesion in less than $50 \%$ of cases [6]; among them, an accidental bite or repeated injury leads to the introduction of viral or toxic agents in the tissues causing inflammatory response [7,8] and local immune reaction [9]. However, an absolute linkage between injuries and the development of these lesions wasn't found in all studies $[10,11]$.

Clinically ulcer appears solitary with elevated and indurated margins associated with pain in many cases. It can persist for several weeks or months without treatment and generally tends to resolve spontaneously [12].

It affects a wide age range of patients from childhood to old age with a peak incidence between the $6^{\text {th }}$ and $7^{\text {th }}$ decades of life and a slight female predominance. The ventral or lateral surface of the tongue is generally involved perhaps because is more vulnerable to injuries. Other oral areas like lip, palate and vestibular mucosa may also be involved. In our case, TUGSE presented in a female child atypically on the left dorsal surface of the tongue measuring about $2 \mathrm{~cm} \times 1.5 \mathrm{~cm}$, tender on palpation with indurated margins surrounded by hyperemic area. Dentoscheletrical system has presented no anomalies. A traumatic episode of an accidental bite referred by the patient a week ago has not justified completely the entity of lesion. No lymphadenopathy was founded. Negative results of laboratory exams excluded a systemic condition, and then biopsy was recommended to define the diagnosis.

Indeed benign nature of lesion has been confirmed by histopathological findings. Microscopical examination of our lesion revealed a polymorphic inflammatory infiltrate mainly composed of eosinophils and histiocytes CD68+, accompanied by a population of $\mathrm{CD} 3+$ lymphocytes $\mathrm{T}$, CD20+ lymphocytes B and macrophages [10] with abundant cytoplasm, irregular nuclear contours, small nucleoli and fine chromatin [11]; immunohistochemical technique results consisted of CD31+ in vascular component and CD30 and S100 rarely expressed. This inflammatory infiltrate extends from the superficial mucosa to the submucosa involving muscle fibers and sometimes salivary glands. The role of eosinophils is not completely clear because they are not present in all traumatic oral ulcers; they may be involved in a tissue reaction to some unknown antigen introduced through the traumatic lesion. Degeneration of oral mucosa may be attributed to the proliferation of cytotoxic $\mathrm{T}$ cells or toxic product released by degranulating eosinophils. It supports the role of cytotoxic T cells in the pathogenesis of TUGSE.

The differential diagnosis is complex because clinical presentation mimics malignancies such as squamous cell carcinoma, lymphoma, salivary gland tumors, infective diseases such as leishmaniasis, toxoplasmosis, hepatitis or autoimmune diseases. The histologic differential diagnosis may include many lesions characterized by infiltration of eosinophils within the connective tissue such as Langerhans cell disease, Angiolymphoid Hyperplasia with Eosinophilia (ALHE), Kimura disease, certain types of lymphomas, allergic reactions and parasitic diseases [12,13]. In our case rarely expressed CD30 antigen excluded a lymphoproliferative disorder [14].

In our patient we observed a marked improvement of the lesion after incisional biopsy with no need for more radical surgery; a complete resolution at 2 and 6 months follow-up visits was observed, indicating a full recovery [15].

\section{Conclusion}

TUGSE is a benign lesion of the oral mucosa of unclear pathogenesis. This case report analyzed clinical and histopathological characteristics of TUGSE highlighting the complexity of diagnosis, due to a large number of pathologies with overlapping clinical and histopathological features. According to literature, some cases show a correlation between TUGSE and recurrent traumatic injury to the tongue. The present case characterized by the young age of the patient, history of an accidental bite and spontaneous selfhealing of the lesion related to the typical clinical picture and histopathological findings led up to the diagnosis of TUGSE. This lesion could be considered a reactive process secondary to trauma excluding other similar suspected ulcerative lesions.

Awareness of this entity is important to emphasize the correct diagnosis of ulcerated lesions and deliver appropriate and effective treatment.

\section{Acknowledgment}

The authors would like to thank the medical staff of the Anatomopathology Department of Magna Graecia University for providing us with detailed histological images and reports used in this study.

\section{References}

1. Soyele O, Adesina O, Ladeji A, Kuye K, Owotade F. Traumatic ulcerative granuloma with stromal eosinophilia: Review of the literature and case report. African Journal of Medical and Health Sciences. 2017; 16: 115

2. Bacem AE Ottoman. Granular cell tumor of the tongue: a case report with emphasis on the diagnostic and therapeutic proceedings. Oncology and Cancer Case Reports. 2015, 1: 1.

3. Riga A. Di una malattia della prima infanzia, Probabilmente non-trattata, di movimenti patologici. Napoli. 1881.

4. Fede F. Della produzione sottolinguale o malattia di Riga. Atto Congresso italiano di pediatria 1890. Napoli. 1891: 251. 
5. Elzay RP. Traumatic ulcerative granuloma with stromal eosinophilia (Riga-Fede's disease and traumatic eosinophilic granuloma). Oral Surgery Oral Medicine Oral Pathology. 1983; 55: 497-506.

6. Segura S, Romero D, Colomo L. Eosinophilic ulcer of the oral mucosa: Another rhistological simulator of $\mathrm{CD} 30+$ lymphoproliferative disorders. British Journal of Dermatology. 2006; 155: 460-63.

7. Gao S, Wang Y, Liu N, Li S, Du J. Eosinophilic ulcer of the oral mucosa: A clinicopathological analysis. Chinese Journal of Dental Research. 2000; 3: 47-50.

8. Hirshberg A, Amariglio N, Akrish S, et al. Traumatic ulcerative granuloma with stromal eosynophilia: Reactive lesion of the oral mucosa. American Journal of Clinical Pathology. 2006; 126: 522-529.

9. El-Mofty SK, Swanson PE, Wick MR, Miller AS. Eosinophilic ulcer of the oral mucosa: Report of 38 new cases with immunohistochemical observations. Oral Surgery Oral Medicine Oral Pathology. 1993; 75: 716-722.

10. Elovic AE, Gallgher GT, Kabani S, Galli SJ, Weller PF, et al. Lack of TGF-a and TGF-b synthesis by human eosinophils in chronic oral ulcers. Oral Surgery Oral Medicine Oral Pathology, Oral Radiology, and Endodontology. 1996; 81: 672-681.

11. Vélez A, Alamillos FJ, Dean A, Rodas J, Acosta A. Eosinophilic ulcer of the oral mucosa: Report of a recurrent case on the tongue. Clinical and Experimental Dermatology.1997; 22: 154-156.

12. Gonçales ES, Rubira-Bullen IF, Rubira CM, Miyazawa M, Chinellato LE, et al. Eosinophilic ulcer of the oral mucosa versus squamous cell carcinoma. Quintessence International. 2007; 38: 677-680.

13. Cepeda LT, Pieretti M, Chapman SF, Horenstein MG. CD30positive atypical lymphoid cells in common on neoplastic cutaneous infiltrate rich in neutrophils and eosinophils. American Journal of Surgical Pathology. 2003; 27: 912-918.

14. Ada S, Seckin D, Tarhan E, Buyuklu F, Cakmak O, et al. Eosinophilic ulcer of the tongue. Australasian Journal of Dermatology. 2007; 48: 248-250.

15. Sharma B, Koshy G, Kapoor S. Traumatic ulcerative granuloma with stromal eosinophilia: A case report and review of pathogenesis. Journal of Clinical and Diagnostic Research. 2016; 10: ZD07-Zd09. 